\title{
Erratum: High Energy Electron Beams from a Laser Wakefield Acceleration with a Long Gas Jet
}

\author{
[J. Korean Phys. Soc. 71, 256 (2017)] \\ Jaehoon $\mathrm{KIM}^{*}$ and Yong Hun HwangBO \\ Center for EM waves, Korea Electrotechnology Research Institute, Ansan 15588, Korea \\ Shin-Yeong LEE \\ Accelerator and nuclear fusion physical engineering, \\ University of Science and Technology, Daejeon 34113, Korea
}

DOI: $10.3938 / \mathrm{jkps} .71 .592$

One affiliation of Shin-Yeong Lee was omitted. It should be added as "Radiation Center for Ultrafast Science, Korea Atomic Energy Research Institute, Daejeon 34057, Korea".

*E-mail: jkim@keri.re.kr; Fax: +82-31-8040-4189 